\title{
PET/CT and breast cancer subtypes
}

\author{
Laura Gilardi • Marco Colleoni • Giovanni Paganelli
}

Published online: 8 June 2013

(C) Springer-Verlag Berlin Heidelberg 2013

Breast cancer is a profoundly heterogeneous disease that includes a number of distinct entities with specific pathological features, biological behaviours and different sensitivities to systemic and targeted therapies [1-4].

The recent development of high-throughput molecular methods offers new opportunities to capture the wide range of this variability. Indeed, the novel molecular techniques of gene expression profiling permit evaluation of the expression of thousands of genes in a large number of tumours, so that a fully comprehensive portrait or profile of the different molecular pathways can be obtained. The reproducibility and the clinical usefulness of assays based on gene expression have been demonstrated with increasing confidence over the past decade, but cost considerations limit the wide availability of these techniques [5]. Immunohistochemical (IHC) determination of oestrogen and progesterone receptors (ER and PgR), the detection of overexpression and/or amplification of human epidermal growth factor receptor 2 (HER2) and the Ki67 labelling index have been defined as a convenient alternative to molecular subtyping and are considered a sufficient to guide therapeutic choices [6].

Several studies have investigated the correlation between these clinical/pathological prognostic and predictive markers and the levels of radiotracer uptake by primary breast tumours, measured as maximum standardized uptake value $\left(\mathrm{SUV}_{\max }\right)$, during ${ }^{18}$ F-FDG PET/CT. High SUV $_{\max }$ was found to be significantly correlated with ER negativity, PgR negativity,

\footnotetext{
L. Gilardi $\cdot$ G. Paganelli $(\bowtie)$

Division of Nuclear Medicine, European Institute of Oncology, via Ripamonti 435, 20141 Milan, Italy

e-mail: divisione.medicinanucleare@ieo.it

M. Colleoni

Division of Medical Senology, European Institute of Oncology, Milan, Italy
}

triple negativity and Ki67 values, while conflicting results were found for the relationship with HER2 status [7-12].

In the present issue of the European Journal of Nuclear Medicine and Molecular Imaging, García Vicente et al. take a step forward in this approach to evaluation. They analysed the correlation between the glycolytic characteristics of primary breast tumours and their molecular subtypes. The authors prospectively evaluated 168 patients who were submitted to FDG PET/CT before neoadjuvant therapy for locally advanced breast cancer. Tumour subtypes were classified by IHC surrogates as luminal A, luminal B-HER2(-), luminal B-HER2(+), HER2 (+) or basal (triple negative), following the recommendations of the 12th St Gallen International Breast Cancer Conference. Statistically significant differences were found in semiquantitative metabolic parameters among the different subtypes, with greater values in HER2 $(+)$ and basal tumours [13]. These results are not unexpected, as the relationship between increased glucose metabolism and tumour aggressiveness is well known. This is one of the first studies demonstrating a correlation between molecular and glycolytic phenotypes of breast cancer.

Humbert et al. found that baseline FDG uptake of primary tumours and its early response to neoadjuvant chemotherapy may vary according to the IHC subtype of the breast cancer. Triple-negative tumours showed the highest baseline SUV. After the first course of therapy the decrease in SUV was significantly higher in triple-negative and HER2-positive subtypes. Pathological complete response (pCR) occurred more often in these two subtypes, rather than in luminal tumours, and the relative change in SUV was predictive of pCR only in HER2-positive tumours with an accuracy of $76 \%$ [14]. Zhang et al. evaluated 244 patients with metastatic breast cancer. They found that molecular subtype, visceral metastases and number of organs bearing metastases could be used to predict logarithmic values of $\mathrm{SUV}_{\max }$ only in previously untreated metastatic breast cancer [15]. 
These few studies have led to important suggestions for future PET/CT studies in patients with breast cancer, since this disease cannot be considered as a single entity any longer. First, a clear definition of the different clinical/pathological subtypes is needed in order to allow comparison between studies carried out in different centres and the translation of the results obtained in the clinical management of patients. Moreover, the IHC classification requires the availability of reliable measurements of its individual components, which should be performed in accordance with the published guidelines to minimize errors [16-18]. The assessment of hormone receptors and HER2 status in multicentre study, such as that of Garcia Vicente et al. [13], should be performed in a single, and preferably experienced, laboratory, in order not to affect the statistical validity of the results. Indeed, the false-negative and falsepositive rates in the assessment of these biological parameters are still unacceptably high, as is the intra- and interlaboratory discordance rate. It has been repeatedly shown that the falsenegative rate for ER and PgR may be as high as $20 \%$, whereas the false-positive rate is $2-4 \%$ for ER but much higher, up to $15 \%$, for PgR [19].

We should keep in mind, however, that subtypes defined by clinical/pathological criteria are similar but not identical to intrinsic subtypes. For example, basal-like tumours are often referred to as triple-negative breast cancer because most basallike tumours are negative for ER, PgR and HER2. This is true for about $75 \%$ of these tumours, while the remaining $25 \%$ include all the other molecular subtypes [20]. Moreover, the clinical/pathological criteria used for the definition of subtypes are rapidly changing and might differ in the near future from those used in the present studies. Recent data support a threshold of $20 \%$ or higher as potentially indicative of high Ki67 status. Recent literature data also support the introduction of PgR to optimally categorize luminal A and luminal B tumours. The proposed value of PgR for distinguishing between these two subtypes is more than $20 \%$, according to a recent study in which the fine tuning was done by comparing the multigene expression-based assays across five independent cohorts and IHC-based definition of luminal A and B tumours [21-23].

In the neoadjuvant setting, higher sensitivities and higher rates of pCR to anthracycline-based chemotherapy among basal-like and HER2(+) tumours have been reported. However, these two breast cancer subtypes show a poor prognosis, with high relapse rate in patients who do not achieve pCR [4, 24]. The earlier identification of this subgroup of patients by FDG PET/CT could facilitate the choice of a more effective therapy.

Another point that deserves attention is the use of FDG $\mathrm{PET} / \mathrm{CT}$ in the initial staging of breast cancer. Garcia Vicente et al. [13] found no statistically significant relationship between molecular subtypes and disease stage, although patients with HER2 $(+)$ tumours showed a higher percentage of distant metastases (35\%) compared to patients with the other subtypes (12\%, $18 \%, 15 \%$ and $20 \%$ for luminal A, luminal B-HER2
(-), luminal B-HER2(+) and basal, respectively). Therefore some subtypes could benefit more than others from a baseline FDG PET/CT evaluation. This point should be clarified by way of a targeted prospective study with an appropriate number of patients.

${ }^{18} \mathrm{~F}-\mathrm{FDG}$ is not the only radiopharmaceutical available to evaluate patients with breast cancer. Novel PET tracers already tested in humans, such as ${ }^{18} \mathrm{~F}$-fluoroestradiol (FES, that binds to ER), ${ }^{18}$ F-FFNP (a progesterone analogue) and ${ }^{68} \mathrm{Ga}-\mathrm{ABY}-002$ and ${ }^{89} \mathrm{Zr}$-trastuzumab (molecular imaging agents with high specificity and affinity for HER2), may provide additional useful information about breast cancer marker expression, tumour heterogeneity and responsiveness to therapy [25-28].

The use of FES PET could have the advantage of allowing in vivo evaluation of ER expression and functional status, and bypasses the possibility of an error in the pathological determination of this molecular marker. Linden et al. demonstrated that the degree of FES uptake has the ability to predict response to endocrine therapy: low or absent FES uptake can identify patients unlikely to obtain an objective response and can lead to the exclusion of an ineffective treatment in patient management [29]. We can also hypothesize that the presence of FES uptake in triple-negative breast cancer could in vivo identify non-basal-like subtypes, that can show ER expression, even if decreased, as reported by Bidard et al. [30]. These patients could therefore be treated with targeted endocrine therapies, which would otherwise be denied.

Finally, both FDG and non-FDG tracers could be useful in the evaluation of metastatic breast cancer. Metastases may display characteristics that do not match those of the primary tumours, with discordance rates reaching $40 \%$ for ER status [31]. Clinical management of disease must therefore adapt to the changing and evolving nature of the tumour over time. The reassessment of breast cancer molecular markers has proved useful in the management of relapsed disease, leading to a change in planned therapy in a significant percentage of patients [31,32]. However, not all metastatic sites can be easily accessible to biopsy, and the results can be inconclusive in a proportion of patients. Histopathological evaluation of a single site of disease is therefore not representative of the global state of marker expression. In contrast, PET/CT is a total-body procedure and provides information about all sites of relapse in a single test.

In conclusion, combined evaluation of a patient by PET/CT scanning with different tracers could permit the outlining of a portrait of breast cancer heterogeneity. Thus, clinicians could have the tools to choose the best treatment option at each stage, to evaluate response to each therapy, to better characterize the prognosis of patients and to monitor the evolution of the disease. A noninvasive, personalized determination of glycolytic activity and molecular marker expression may therefore become the basis for treatment of individual patients with different subtypes of breast cancer. 


\section{References}

1. Perou CM, Sørlie T, Eisen MB, van de Rijn M, Jeffrey SS, Rees $\mathrm{CA}$, et al. Molecular portraits of human breast tumours. Nature. 2000;406:747-52.

2. Sørlie T, Perou CM, Tibshirani R, Aas T, Geisler S, Johnsen H, et al. Gene expression patterns of breast carcinomas distinguish tumor subclasses with clinical implications. Proc Natl Acad Sci U S A. 2001;98:10869-74.

3. Kennecke H, Yerushalmi R, Woods R, Cheang MCU, Voduc D, Speers $\mathrm{CH}$, et al. Metastatic behaviour of breast cancer subtypes. J Clin Oncol. 2010;28:3271-7.

4. Carey LA, Dees EC, Sawyer L, Gatti L, Moore DT, Collichio F, et al. The triple negative paradox: primary tumor chemosensitivity of breast cancer subtypes. Clin Cancer Res. 2007;13:2329-34.

5. Prat A, Ellis MJ, Perou CM. Practical implications of geneexpression-based assays for breast oncologists. Nat Rev Clin Oncol. 2012;9:48-57.

6. Goldhirsch A, Wood WC, Coates AS, Gelber RD, Thürlimann B, Senn H-J, et al. Strategies for subtypes - dealing with the diversity of breast cancer: highlights of the St. Gallen International Expert Consensus on the Primary Therapy of Early Breast Cancer 2011. Ann Oncol. 2011;22:1736-47.

7. Kim BS, Sung SH. Usefulness of 18F-FDG uptake with clinicopathologic and immunohistochemical prognostic factors in breast cancer. Ann Nucl Med. 2012;26:175-83.

8. Tchou J, Sonnad SS, Bergey MR, Basu S, Tomaszewski J, Alavi A, et al. Degree of tumor FDG uptake correlates with proliferation index in triple negative breast cancer. Mol Imaging Biol. 2010;12:657-62.

9. Basu S, Chen W, Tchou J, Mavi A, Cermik T, Czerniecki B, et al. Comparison of triple-negative and estrogen receptor-positive/progesterone receptor-positive/HER2-negative breast carcinoma using quantitative fluorine-18 fluorodeoxyglucose/positron emission tomography imaging parameters: a potentially useful method for disease characterization. Cancer. 2008;112:995-1000.

10. Groheux D, Giacchetti S, Moretti J-L, Porcher R, Espié M, Lehmann-Che J, et al. Correlation of high 18F-FDG uptake to clinical, pathological and biological prognostic factors in breast cancer. Eur J Nucl Med Mol Imaging. 2011;38:426-35.

11. Sanli Y, Kuyumcu S, Ozkan ZG, Ișık G, Karanlik H, Guzelbey B, et al. Increased FDG uptake in breast cancer is associated with prognostic factors. Ann Nucl Med. 2012;26:345-50.

12. Ueda S, Tsuda $H$, Asakawa $H$, Shigekawa $T$, Fukatsu $K$, Kondo N, et al. Clinicopathological and prognostic relevance of uptake level using 18F-fluorodeoxyglucose positron emission tomography/computed tomography fusion imaging (18FFDG PET/CT) in primary breast cancer. Jpn J Clin Oncol. 2008:38:250-8.

13. García Vicente AM, Soriano Castrejón A, León Martín A, Chacón López-Muñiz I, Muñoz Madero V, Del Mar Muñoz Sánchez M, et al. Molecular subtypes of breast cancer: metabolic correlation with $18 \mathrm{~F}$ FDG PET/CT. Eur J Nucl Med Mol Imaging. 2013. doi:10.1007/ s00259-013-2418-7.

14. Humbert O, Berriolo-Riedinger A, Riedinger JM, Coudert B, Arnould L, Cochet A, et al. Changes in 18F-FDG tumor metabolism after a first course of neoadjuvant chemotherapy in breast cancer: influence of tumor subtypes. Ann Oncol. 2012;23:2572-7.

15. Zhang J, Jia Z, Zhou M, Ragaz J, Zhang Y-P, Wang B-Y, et al. The SUVmax for 18F-FDG correlates with molecular subtype and survival of previously untreated metastatic breast cancer. Clin Nucl Med. 2013;38:256-62.
16. Hammond ME, Hayes DF, Dowsett M, Allred DC, Hagerty KL, Badve $\mathrm{S}$, et al. American Society of Clinical Oncology/College Of American Pathologists guideline recommendations for immunohistochemical testing of estrogen and progesterone receptors in breast cancer. J Clin Oncol. 2010;28:2784-95.

17. Wolff AC, Hammond ME, Schwartz JN, Hagerty KL, Allred DC, Cote RJ, et al. American Society of Clinical Oncology/College of American Pathologists guideline recommendations for human epidermal growth factor receptor 2 testing in breast cancer. J Clin Oncol. 2007;25:118-45.

18. Dowsett M, Nielsen TO, A'Hern R, Bartlett J, Coombes RC, Cuzick J, et al. Assessment of Ki67 in breast cancer: recommendations from the International Ki67 in Breast Cancer working group. J Natl Cancer Inst. 2011;103:1656-64.

19. Viale G. Integrating molecular profiling, histological type and other variables: defining the fingerprint of responsiveness to treatment Breast. 2009;18:S32-6.

20. Perou CM. Molecular stratification of triple-negative breast cancer. Oncologist. 2011;16:61-70.

21. Nielse TO, Polley M-YC, Leung SCY, et al. An international Ki-67 reproducibility study. Presented at the 35th San Antonio Breast Cancer Symposium, 4-8 December 2012 (abstract S4-6).

22. Prat A, Cheang MC, Martin M, Parker JS, Carrasco E, Caballero R, et al. Prognostic significance of progesterone receptor-positive tumor cells within immunohistochemically defined luminal A breast cancer. J Clin Oncol. 2013;31:203-9.

23. de Azambuja E, Cardoso F, de Castro G, Colozza Jr M, Mano MS, Durbecq V, et al. Ki-67 as prognostic marker in early breast cancer: a meta-analysis of published studies involving 12,155 patients. $\mathrm{Br} \mathrm{J}$ Cancer. 2007;96:1504-13.

24. Rouzier R, Perou CM, Symmans WF, Ibrahim N, Cristofanilli M, Anderson K, et al. Breast cancer molecular subtypes respond differently to preoperative chemotherapy. Clin Cancer Res. 2005;11:5678-85.

25. Sundararajan L, Linden HM, Link JM, Krohn KA, Mankoff DA. 18F-Fluoroestradiol. Semin Nucl Med. 2007;37:470-6.

26. Dehdashti F, Laforest R, Gao F, Aft RL, Dence CS, Zhou D, et al. Assessment of progesterone receptors in breast carcinoma by PET with $21-18 \mathrm{~F}$-fluoro-16 $\alpha, 17 \alpha$-[(R)-(1'- $\alpha$-furylmethylidene)dioxy]-19norpregn-4-ene-3,20-dione. J Nucl Med. 2012;53:363-70.

27. Baum RP, Prasad V, Müller D, Schuchardt C, Orlava A, Wennborg $\mathrm{A}$, et al. Molecular imaging of HER2-expressing malignant tumors in breast cancer patients using synthetic $111 \mathrm{In}$ - or $68 \mathrm{Ga}$-labeled Affibody molecules. J Nucl Med. 2010;51:892-7.

28. Dijkers EC, Oude Munnink TH, Kosterink JG, Browers AH, Jager PL, de Jong JR, et al. Biodistribution of 89Zr-trastuzumab and PET imaging of HER2-positive lesions in patients with metastatic breast cancer. Clin Pharmacol Ther. 2010;87:586-92.

29. Linden HM, Stekhova SA, Link JM, Gralow JR, Livingston RB, Ellis GK, et al. Quantitative fluoroestradiol positron emission tomography imaging predicts response to endocrine treatment in breast cancer. J Clin Oncol. 2006;24:2793-9.

30. Bidard FC, Conforti R, Boulet T, Michiels S, Delaloge S, André F. Does triple-negative phenotype accurately identify basal-like tumour? An immunohistochemical analysis based on 143 'triplenegative' breast cancers. Ann Oncol. 2007;18:1285-6.

31. Simmons C, Miller N, Geddie W, Gianfelice D, Oldfield M, Dranitsaris G, et al. Does confirmatory tumor biopsy alter the management of breast cancer patients with distant metastases? Ann Oncol. 2009;20:1499-504.

32. Curigliano G, Bagnardi V, Viale G, Fumagalli L, Rotmensz N, Aurilio G, et al. Should liver metastases of breast cancer be biopsied to improve treatment choice? Ann Oncol. 2011;22:2227-33. 